\title{
Natural Products into Drugs for the Treatment of Neurodegenerative Diseases
}

\section{Burgos EG}

Department of Pharmacology, University Complutense Madrid, Spain

*Corresponding author: Burgos EG, Department of Pharmacology, University Complutense Madrid, Spain, Tel: 91394 2276; E-mail: elenagon@ucm.es Received date: February 20, 2017; Accepted date: February 23, 2017; Published date: February 27, 2017

Copyright: () 2017 Burgos EG. This is an open-access article distributed under the terms of the Creative Commons Attribution License, which permits unrestricted use, distribution, and reproduction in any medium, provided the original author and source are credited.

\section{Editorial}

Neurodegenerative diseases such as Parkinson's disease, Alzheimer's disease, Huntington's disease, and amyotrophic lateral sclerosis constitute a great problem of public health and one of the major medical challenges. These diseases are characterized by the progressive loss of the structural and functional features of neurons and as consequence are produced serious physical effects, and emotional, social behaviour and cognitive disturbances. Moreover, these central nervous system disorders share the characteristics that their prevalence and incidence increase with age and up to date they are incurable (current available treatment is symptomatic). Neurodegenerative diseases are found among the most devastating pathologies, bringing human suffering (for the patient himself, their family and caregivers) and a heavy economic burden.

Natural products from plants, marines, animals, fungal and bacterial are known and employed since ancient times for their therapeutic properties. Considerable numbers of the medicines currently on the market are of natural origin or they are synthetic and semisynthetic drug products based on natural product pharmacophores. Polyphenols, terpenes and alkaloids are found among the most studied and promising potential drugs for the prevention and treatment of age-associated neurodegenerative diseases mainly due to their antioxidant, autophagy modulation, antiinflammatory, mitochondrial function modulation and antiamyloidogenic properties.

We invite to contribute with in vivo and in vitro basic research articles, review articles and clinical studies regarding the role of natural products as leads to potential drugs for current age-associated neurodegenerative diseases treatment and the outlook for advanced medicinal therapies. 